\title{
Nuclear Binding Energies and NN uncertainties
}

\section{Rodrigo Navarro Perez ${ }^{* \dagger}$}

Departamento de Física Atómica, Molecular y Nuclear and Instituto Carlos I de Física Teórica y Computacional. Universidad de Granada, E-18071 Granada, Spain.

E-mail: rnavarrop@ugr.es

\section{J. E. Amaro}

Departamento de Física Atómica, Molecular y Nuclear and Instituto Carlos I de Física Teórica y Computacional. Universidad de Granada, E-18071 Granada, Spain.

E-mail: amaro@ugr.es

\section{E. Ruiz Arriola}

Departamento de Física Atómica, Molecular y Nuclear and Instituto Carlos I de Física Teórica y Computacional. Universidad de Granada, E-18071 Granada, Spain.

E-mail: earriola@ugr.es

There is an increasing interest in quantifying the predictive power in nuclear structure calculations. We discuss how both experimental and systematic errors at the NN-level can be used to estimate the theoretical uncertainties by rather simple means and without solving the full nuclear many body problem. We emphasize the role of effective interactions defined by coarse graining the NN potential to length scales of the order of the minimal de Broglie wavelength probed between nucleons in nuclei. We find an a priori error of $\Delta B / A \sim 0.1-0.4 \mathrm{MeV}$ for the binding energy per particle throughout the periodic table for $2 \leq A \leq 208$, and a linear growth of the error with density for nuclear matter $\Delta B / A \sim 3.75 \rho_{\mathrm{n} \text {.m. }}$ and neutron matter $\Delta B / N \sim 3.5 \rho_{\mathrm{n}}$. This suggests to limit the computational effort in solving the Nuclear Many Body Problem to such an accuracy.

Sixth International Conference on Quarks and Nuclear Physics

April 16-20, 2012

Ecole Polytechnique, Palaiseau, Paris

\footnotetext{
* Speaker.

${ }^{\dagger}$ Work supported by Spanish DGI (grant FIS2011-24149) and Junta de Andalucía (grant FQM225). R.N.P. is supported by a Mexican CONACYT grant.
} 


\section{Introduction}

The present contribution is based on our recent work [1], where an old issue is adressed from a modern perspective. The key question is, can we quantify errors in binding energies based on our incomplete knowledge of the NN interaction?.

The nuclear many body problem consists of diagonalizing the Hamiltonian $H$ with multinucleon interactions,

$$
H=\sum_{i} T_{i}+\sum_{i<j} V_{2, i j}+\sum_{i<j<k} V_{3, i j k}+\sum_{i<j<k<l} V_{4, i j k l}+\ldots
$$

where the indices run $2 \leq i \leq A$ and $T_{i}=p_{i}^{2} /\left(2 M_{N}\right)$ is the kinetic energy of the $i$-th nucleon, $V_{2, i j}$ the NN potential, $V_{3, i j k}$ the NNN potential, etc. Roughly speaking one can fix $V_{2}$ from the deuteron and $\mathrm{NN}$-scattering data, $V_{3}$ from triton and nucleon-deuteron scattering, $V_{4}$ from the $\alpha$-particle and any scattering process involving four nucleons, etc.

First principles calculations in Nuclear Physics have traditionally been dominated by the idea that once the fundamental NN-interaction is accurately known one is left with the intricacies and complexities of the many body problem on the theoretical side. Actually, ab initio calculations in Nuclear Physics have been carried out as if there was complete knowledge on the elementary NN-dynamics. Therefore, a long-term effort has been carried out to substantiate this assumption by continuously improving the NN-potentials, but little attention has been paid to determine the uncertainty in the potentials themselves. We have recently filled this gap by carefully analyzing different error sources [2] and using the concept of a coarse grained potential [3].

\section{NN potentials}

Along the years many studies have been oriented towards improving the NN-potentials by performing $\chi^{2}$-fit of a Partial Wave Analysis to the abundant available pp and np experimental data. From a purely statistical analysis point of view, we remind that in order to determine reliable confidence levels on the fitting parameters, i.e. error estimates, one must have $\chi^{2} /$ d.o.f $\lesssim 1$. Larger values of the reduced $\chi^{2}$ (most Bonn,Paris, Esc or Nijm93 potentials produced $\chi^{2} /$ d.o.f $\sim 2$ ) would actually diminish the uncertainties, as there is a large penalty to change the most likely fit parameter values, and would produce unreliable error estimates. Thus, an error analysis of NN phase-shifts for several partial waves became first possible when the Nijmegen group [4] carried out a Partial Wave Analysis (PWA) fitting about 4000 experimental np and pp data (after rejecting further 1000 of $3 \sigma$-mutually inconsistent data) with $\chi^{2} /$ dof $\sim 1$.

In general, fits consider differential cross sections, polarization asymmetries, etc. for a given set of energies and angles $\left(E_{i}, \theta_{i}\right)$. While the partial wave expansion allows to evaluate any scattering angle, the fixed energies allow to determine phase shifts only at those measured energies, so that the phase-shifts themselves become independent fitting parameters. On the other hand, the analyticity of the S-matrix based on the meson-exchange picture, guarantees that the phase-shifts are smooth functions for real positive energy. A handy way to combine this energy-angle information and implementing the expected smooth energy dependence is by introducing an auxiliary potential with a set of adjustable parameters. The bench-marking Nijmegen fit fixed the form of the potential to incorporate charge dependence. It contains an energy dependent square well operating at a 
distance below 1.4fm, a One-Pion-Exchange (OPE) contribution starting at $1.4 \mathrm{fm}$, a One-BosonExchange (OBE) piece below 2-2.5fm and an electromagnetic contribution. Energy dependence reflects retardation in the interaction due to unobserved virtual excitations. However, it is inconvenient to perform Nuclear structure calculations since it requires solving a time-dependent many body problem. At present there are a variety of $\mathrm{NN}$ (energy independent) potentials fitting a large body of scattering data with $\chi^{2} /$ dof $\sim 1[4,5,6,7,8]$ which allow the application of conventional stationary dynamics. Surprisingly, error estimates on the potential fitting parameters are never provided. In [2] we analyze different error sources, in particular the short distance non-localities of the interaction which may depend on energy, linear and angular momentum. While in principle these non-localities are on-shell equivalent (see e.g. Ref. [9] for a proof in a $1 / M_{N}$ expansion) they generate quantitative differences.
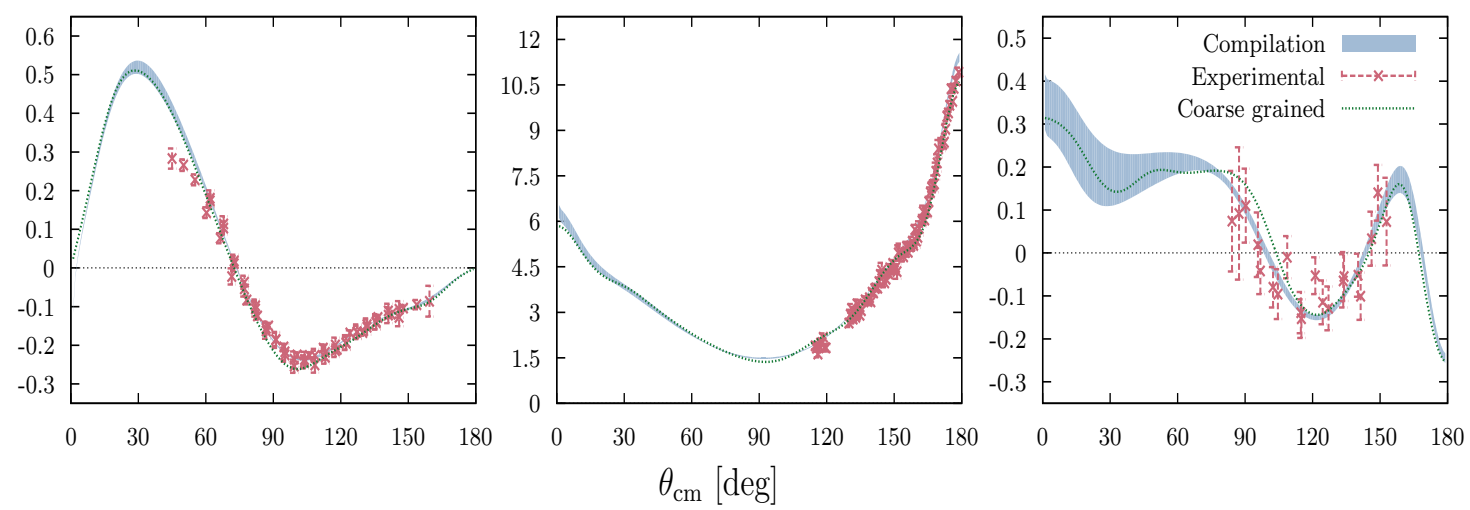

Figure 1: np scattering observables for different energies in the laboratory system as a function of the CM angle. Left panel: Polarization at $E_{\mathrm{LAB}}=325 \mathrm{MeV}$. Middle panel: Differential cross section at $E_{\mathrm{LAB}}=$ 324.1MeV. Right panel: $D_{t}$ depolarization at $E_{\mathrm{LAB}}=325 \mathrm{MeV}$. The short-dashed line denotes our results by the coarse grained interaction [2]. The band represents the compilation of the PWA and five high quality potentials $[4,6,7,8]$ which provided a $\chi^{2} /$ d.o.f $\lesssim 1$. For references for the experimental data see http://nnonline.org and http://gwdac.phys.gwu.edu/.

From our reanalysis of the discrepancies in the different partial waves we have found that the PWA statistical errors quoted in the original Nijmegen work [4], $\Delta \delta_{\mathrm{PWA}}$, turn out to be smaller than the discrepancies among the fitted phases stemming from the different potential fits, separately claiming a $\chi^{2} /$ d.o.f. $\lesssim 1$ to data. In other words,

$$
\Delta \delta_{\mathrm{PWA}} \lesssim \sqrt{\frac{1}{N-1} \sum_{n}\left(\delta_{n}-\bar{\delta}\right)^{2}}, \quad \delta_{n}=\delta_{\mathrm{NijmII}}, \delta_{\mathrm{Reid} 93}, \delta_{\mathrm{NijmI}}, \delta_{\mathrm{Av} 18}, \delta_{\mathrm{Spec}}, \delta_{\mathrm{CDBonn}}
$$

Thus at $E_{\mathrm{LAB}}=350 \mathrm{MeV}$ in the ${ }^{1} S_{0}$ channel one has a s.d. of $0.7^{\circ}$ whereas $\Delta \delta_{\mathrm{PWA}}=0.3^{0}$. This counter-intuitive result relies not only on the specific forms of potentials which treat the mid- and short-range behaviour of the interaction differently but also on the fact that the fits are mainly done to scattering amplitudes rather than to the phase-shifts themselves. The systematic discrepancies are vividly illustrated by looking at Fig. 1 where the spread of the different potentials and the PWA analysis increases in the region where no data constrain the result. 


\section{Direct error estimates}

The most direct way of quantifying binding energy uncertainties would be to undertake large scale $a b$ initio calculations using the different two-body potentials, say $V_{2}^{(i)}$ with $i=1, \ldots N$, yielding $B^{(i)}(A)$ whence a mean $\bar{B}(A)$ and a standard deviation $\Delta B(A)$ can be constructed,

$$
\bar{B}(A)=\frac{1}{N} \sum_{i} B^{(i)}(A) \quad \Delta B(A)=\sqrt{\frac{1}{N-1} \sum_{i}\left(B^{(i)}(A)-\bar{B}(A)\right)^{2}}
$$

For instance, the triton binding energy obtained by Faddeev calculations is 8.00, 7.62, 7.63, 7.62, 7.72 and $8.50 \mathrm{MeV}$ for the CD Bonn [10], Nijm-II, Reid93, Nijm-I, AV18 [11] and the covariant spectator model [8] respectively. This yields the combined result $B_{3}=7.85(34) \mathrm{MeV}$ (exp. $B_{3}=$ $8.4820(1) \mathrm{MeV}$ ) i.e. $\Delta B_{3} / 3=0.11 \mathrm{MeV}$. Note that a three-body interaction would account for the missing $1 \mathrm{MeV}$-binding.

This error estimate procedure stops beyond the $A=4$ nucleus, due to computational and theoretical difficulties related to the form of the potential. From an ab initio viewpoint, only Monte Carlo calculations may go up to $A=10$ when potentials are fixed to be $r$-dependent with a nonlocality in terms of the relative angular momentum operator, becoming the standard Hamiltonians for $a b$ initio calculations of light nuclei [12] and dense matter [13]. Again, a lack of error estimate of the potential parameters makes it impossible to deduce the theoretical errors in such calculations. On top of that, one should add the systematic errors due to the different forms of the potential.

Finite experimental accuracy provides also a statistical error $\Delta V_{n}$ on the n-body force. In addition, unless the most general possible n-body forces are considered some systematic error is introduced. On the other hand, the definition of the three- and higher-body interaction depends on the two body potential, so any uncertainty in the two-body interaction will carry over to the three-body interaction. Thus, even if we fix it say in the $A=3$ system, there will always be a residual uncertainty in the $A+1=4$ calculation. To fix ideas it is convenient to think of the $\mathrm{n}$ body potentials as random variables. They depend on some unknown parameters $\left(c_{1}, \ldots c_{N}\right)$ and will eventually be determined from $\chi^{2}$-fit to some data involving n-body interactions. This would provide a probability distribution of parameters $P\left(c_{1}, \ldots c_{N}\right)$, according to which the normalized variable $v_{n}=\left(V_{n}-\left\langle V_{n}\right\rangle\right) / \sqrt{\left\langle\left(V_{n}-\left\langle V_{n}\right\rangle\right)^{2}\right\rangle}$ which has zero mean, $\left\langle v_{n}\right\rangle=0$, and unit variance, $\Delta v_{n}=$ 1 , can be defined. Correlations between the two- and three-body forces can be ignored within errors if $\left(\left\langle v_{2} v_{3}\right\rangle\right)^{2} \lesssim\left(\left\langle\left(v_{2} v_{3}-\left\langle v_{2} v_{3}\right\rangle\right)^{2}\right\rangle\right.$. Although establishing the validity of this condition requires a thourough analysis of multinucleon forces, we will assume this to be the case, so that

$$
\Delta B(A)^{2}=\Delta V_{2}^{2}+\Delta V_{3}^{2}+\Delta V_{4}^{2}+\ldots
$$

in which case, estimating the two-body uncertainty provides a lower bound on the total uncertainty. Note that non-vanishing statistical correlations may change this.

\section{Error estimates from Coarse grained interactions}

In our recent work [2] we have shown how coarse grained interactions [3] can advantageously be used for the purpose of error estimate. Besides making accurate fits to the combined NNscattering database obtained from the PWA and the 6 high-quality potentials this representation 
allows to side-step the short distance complications arising in nuclear structure calculations. This is very much in spirit of the $V_{\text {lowk }}$-approach [14], the Unitary Correlation Method (UCOM) [15] or the Similarity Renormalization Group (SRG) (see e.g. [16] and references therein), where smooth soft-core potentials are produced after the original potential has been evolved to the relevant scale. However, these transformations are applied to the existing high-quality potentials and become computationally cumbersome. We suggest instead to determine the effective coarse grained interactions directly from fits to the scattering data up to a given maximum energy. For light nuclei $A \leq 40$ it turns out that a reasonable maximum $\mathrm{CM}$ momentum is about $p_{\mathrm{CM}} \sim 200 \mathrm{MeV}$ [3] since the highest wavelength resolution $1 / p \sim 1 \mathrm{fm}$ is much larger than the short range repulsion distance, $a_{\text {core }} \sim 0.5 \mathrm{fm}$, present in central waves. Thus, we expect mean field calculations to be applicable.

In this way the systematic uncertainties due to the different forms of the potential can be propagated. We have found that for doubled-closed shell nuclei S-waves dominate the uncertainty. Using Harmonic Oscillator Shell model wave functions we get [1]

$$
\frac{\Delta B_{3^{3}}}{3}=0.08(1), \quad \frac{\Delta B^{4} \mathrm{He}}{4}=0.12(1), \quad \frac{\Delta B_{{ }^{16} \mathrm{O}}}{16}=0.28(2), \quad \frac{\Delta B^{40} \mathrm{Ca}}{40}=0.34(2) \mathrm{MeV}
$$

the errors depending on the fitting cut-off LAB energy. While one may reasonably doubt that variations in the binding energies can be monitored by coarse grained interactions with harmonic oscillator wave functions, we have found [1] that this is not the case by analyzing variations of binding energies in ab initio calculations induced by a relative $1 \%$ change in the AV18 potential parameters [17].

For heavier nuclei a simple estimate of errors due to the two body interaction uncertainty can be made by using Skyrme effective interactions (for a review see [18]) where for symmetric $N=Z=A / 2$ nuclei one has

$$
\frac{\Delta B}{A}=\frac{3}{8 A} \Delta t_{0} \int d^{3} x \rho(x)^{2},
$$

where $t_{0}=\int d^{3} x\left(V_{{ }^{1} S_{0}}+V_{{ }^{3}}\right) / 2$ and $x_{0} t_{0}=\int d^{3} x\left(-V_{1} S_{0}+V_{{ }^{3} S_{1}}\right) / 2$. Propagating errors we get $t_{0}=$ $-0.92(1) \mathrm{GeVfm}^{3}$, in agreement with the equation of state used by the Trento group [19] at low densities, $t_{0} \sim-0.9(1) \mathrm{GeVfm}^{3}$ and with coarse graining of $\mathrm{NN}$ interactions in $\mathrm{CM}$ momentum space down to $\Lambda \sim 0.3 \mathrm{GeV}$ gives a compatible value, $t_{0} \sim-4 \pi^{2} /\left(M_{N} \Lambda\right)$ [20]. We may implement finite size effects by using a Fermi-type shape for the matter density $\rho(r)=\rho_{0} /\left(1+e^{(r-R) / a}\right)$ with $R=r_{0} A^{\frac{1}{3}}$ and $r_{0}=1.1 \mathrm{fm}$ and $a=0.7 \mathrm{fm}$ and normalized to the total number of particles $A=\int d^{3} x \rho(x)$ we get a result compatible with Eq. (4.1) and depending on the value of $A$ for $4 \leq A \leq 208$. In Fig. (2) we illustrate the situation by imposing our error band on the binding energy of stable nuclei. For nuclear and neutron matter the effect grows linearly with the density (in $\mathrm{fm}^{-3}$ )

$$
\frac{\Delta B_{\text {n.m. }}}{A}=\frac{3}{8} \Delta t_{0} \rho \sim 3.75 \rho, \quad \frac{\Delta B_{n}}{N}=\frac{1}{4} \Delta\left[t_{0}\left(1-x_{0}\right)\right] \rho_{n} \sim 3.5 \rho_{n}
$$

In Fig. 2 we implement our error estimates on the EOS calculations of the Trento group [19], where their uncertainties reflect the accuracy in solving the many body problem only. As we see, their errors are much smaller than those estimated here. We remind that among other effects we have neglected possible statistical correlations between $3 N$ and $2 N$ forces, thus a more thorough analysis incorporating n-body coarse grained interactions would provide a more definite answer. 

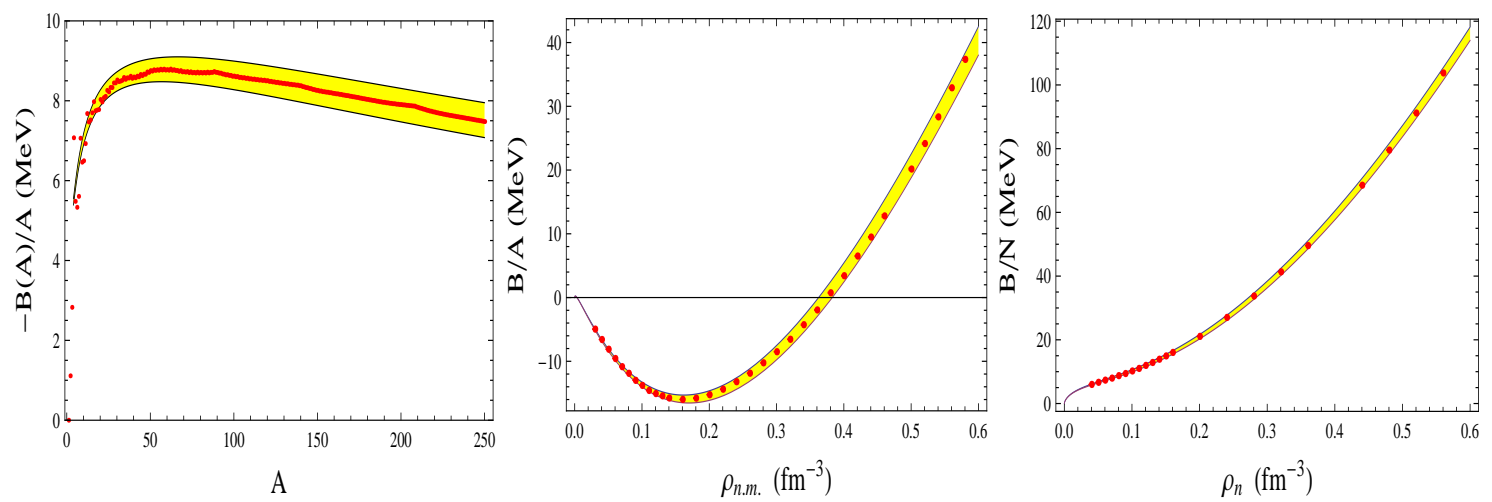

Figure 2: Superposition of estimated errors based on propagating Nucleon-Nucleon uncertainties via coarse grained interactions. Left panel: Stable nuclei. Middle panel: Nuclear Matter Equation of State (EOS). Right Panel: Neutron Matter Equation of State. EOS Data are from the Green Function MonteCarlo Calculation of the Trento group [19].

\section{References}

[1] R. Navarro Perez, J. E. Amaro and E. Ruiz Arriola, arXiv:1202.6624 [nucl-th].

[2] R. N. Perez, J. E. Amaro and E. Ruiz Arriola, arXiv:1202.2689 [nucl-th].

[3] R. Navarro Perez, J.E. Amaro, and E. Ruiz Arriola. Prog.Part.Nucl.Phys., 67:359-364, 2012.

[4] V.G.J. Stoks, R.A.M. Kompl, M.C.M. Rentmeester, and J.J. de Swart. Phys.Rev., C48:792-815, 1993.

[5] V.G.J. Stoks, R.A.M. Klomp, C.P.F. Terheggen, and J.J. de Swart. Phys.Rev., C49:2950-2962, 1994.

[6] Robert B. Wiringa, V.G.J. Stoks, and R. Schiavilla. Phys.Rev., C51:38-51, 1995.

[7] R. Machleidt. Phys.Rev., C63:024001, 2001.

[8] Franz Gross and Alfred Stadler. Phys.Rev., C78:014005, 2008.

[9] A. Amghar and B. Desplanques. Nucl.Phys., A585:657-692, 1995.

[10] R. Machleidt, F. Sammarruca, and Y. Song. Phys. Rev., C53:1483-1487, 1996.

[11] J. L. Friar, G. L. Payne, V. G. J. Stoks and J. J. de Swart, [nucl-th/9307010].

[12] Steven C. Pieper and Robert B. Wiringa. Ann.Rev.Nucl.Part.Sci., 51:53-90, 2001.

[13] A. Akmal and V.R. Pandharipande. Phys.Rev., C56:2261-2279, 1997.

[14] S.K. Bogner, R.J. Furnstahl, and A. Schwenk. Prog.Part.Nucl.Phys., 65:94-147, 2010.

[15] Thomas Neff and Hans Feldmeier. Nucl.Phys., A713:311-371, 2003.

[16] V. S. Timoteo, S. Szpigel and E. R. Arriola, arXiv:1108.1162 [nucl-th].

[17] V.V. Flambaum and Robert B. Wiringa. Phys.Rev., C76:054002, 2007.

[18] Michael Bender, Paul-Henri Heenen, and Paul-Gerhard Reinhard. Rev.Mod.Phys., 75:121-180, 2003.

[19] S. Gandolfi, A.Yu Illarionov, S. Fantoni, J.C. Miller, F. Pederiva, et al. Mon.Not.Roy.Astron.Soc., 404:L35-L39, 2010.

[20] E. Ruiz Arriola, arXiv:1009.4161 [nucl-th]. 